\title{
Detección de patología cervical no diagnosticada por citología vaginal mediante cervigrafía
}

\author{
Hernán Urdaneta*
}

\begin{abstract}
RESUMEN: A un total de 105 pacientes ginecológicas entre 18-65 años se les practicó citología vaginal (CV) y cervigrafia, un método fotográfico no invasivo recientemente introducido para screening. Se evaluó de una manera independiente la precisión de la cervigrafía y de la CV para detectar enfermedad premaligna y maligna del cerviz. Los resultados, cuando fueron positivos, se compararon con el diagnóstico histológico a partir de biopsias dirigidas por colposcopia. No se encontró diferencia significativa en la sensibilidad ( $p=0.2483)$ ni en la especificidad ( $p=0.0721$ ) de ambas pruebas, aunque se detectaron más casos de infección por virus del papiloma humano mediante cervigrafía que mediante $\mathrm{CV}$.
\end{abstract}

El uso simultáneo de la CV y la cervigrafía incrementa la detección de lesiones de bajo y alto grado, disminuye el número de colposcopias que habría que realizar, permite disminuir la frecuencia de la toma de $\mathrm{CV}$, compensando así con creces el costo adicional de la cervigrafía.

PALABRAS CLAVES: Cervigrafía, citología vaginal.

SUMMARY: Over 105 gynecology patients, between 18-65 yearsold, 17 realizeds vaginal; smear and cervigraphy a new phatographic method non invasive for scraning. We took and independent mescice form each one of there methods like predictor of premaling and maling disease of the cervix. The positive results were compare with biopses taking by colcoscopy. It didn't find significative difference on specify and sensibility of the probes al throught, we detected mone infection by human papilomas virus; by cervicography.

The simultaneous use of those two methods improbe the detection of the different leuonson the cervix, disminish the number of colposcopies and the frecuency of vaginal smears.

KEY WORDS: Cervigraphy, Vaginal, Smear

\section{Introducción}

Aunque las lesiones premalignas y malignas del cérvix pueden ser detectadas ampliamente mediante el uso de la citología vaginal (CV), este método no es el ideal ya que presenta una tasa de falsos negativos del orden del 6-55\% (1-4). Adicionalmente la CV clase II de Papanicolaou (Pap), extendido atípico negativo para células malignas, está asociada con entidades clínicamente importantes como la infección por el virus del papiloma humano (VPH) (5), neoplasia intraepitelial cervical (NIC) (6-8) y aún cáncer invasivo (9). La tasa de CV clase II ocurre con una frecuencia informada en la literatura internacional del orden del 1.6-5.4\% (10) existiendo informes de tasas del $29 \%$ (11). En Bogotá se encuentran tasas del orden del $70 \%$.

Una estrategia para mejorar la detección de la patología cervical puede ser repetir la CV. La CV repetida predice la presencia de lesiones de bajo grado en el $53 \%$ de los casos y de alto grado en el $71 \%$ de los casos (21). Se ha sugerido también el empleo de colposcopia en todas las pacientes con CV clase II persistente, pero el volumen de pacientes a evaluar puede ser inmanejable (13). La tipificación de los

* Ginecólogo Obstetra. Profesor Asistente. Sección de Obstetricia y Ginecología Hospital Militar Central. Departamento de Ciencias Fisiológicas Universidad Nacional. Bogotá. genotipos del VPH ha sido mencionada como alternativa para screening de la patología premaligna del cérvix (19) En el medio colombiano esto no tiene especial utilidad debido a que existe una alta prevalencia combinada de los genotipos de mayor riesgo oncogénico (18).

En 1981 Stalf(14) introdujo la cervigrafía o cervicografía con el objeto de mejorar la sensibilidad de los métodos de screening de la enfermedad premaligna y maligna del cérvix a ácido acético al 4-5\% y tomarle dos macrofotografías. Después de revelada la película, el cervigrama (diapositiva) proyectado en una pantalla es comparable a la magnificación y resolución colposcópica. Es numerosa la literatura disponible sobre cervigrafía considerandose que su empleo combinado con la CV mejora la tasa de detección de la patología cervical (24), disminuyendo el costo por caso positivo detectado en relación al empleo de la CV aislada (15).

En el presente estudio se compara la citología vaginal con el cervigrama para detectar el NIC y la infección subclínica por papilomavirus.

\section{Material y métodos}

Se incluyeron 112 pacientes no embarazadas consultantes al Hospital Militar Central, Bogotá, con edades entre 19-65 años a quienes se informó del procedimien- 
to y se solicitó autorización para participar en el estudio. De estas 112 pacientes, 80 consultaron a la clínica de ginecología y 32 a la de colposcopia. A las participantes procedentes de ginecología se les practicó CV. Las consultantes a colposcopia tenían CV tomada 3-4 semanas antes Pap II con atipias coilocíticas (Pap IIAC), Pap III displasia leve (DL), moderada (DM), severa (DS), o Pap IV. A todas las pacientes incluidas en el estudio les fue tratado el cérvix con dos aplicaciones de ácido acético al 4-5\% (25). Se identificó la paciente con una marquilla de acetato colocada dentro del área a fotografiar, y se tomaron dos fotografías (cervigramas) empleando un equipo fotográfico igual al propuesto por Stalf (14). Luego se trato el cérvix con solución de lugol y se tomó una tercera foto.

Los cervigramas se clasificaron de acuerdo con criterios modificados de los originales de Stalf (14): 1- Negativo; Ausencia de lesiones anormales, unión escamocolumnar visible por lo menos en un $85 \%$ y zona de transformación (ZT) visibles en su totalidad. 2- Atípico: Sugestivo de infección por papiloma virus fuera de la ZT, metaplasia escamosa inmadura atípica. Cambios triviales de dudosa interpretación. 3- Positivo: Epitelio acetoblanco, punteado, mosaico, vasos atípicos. 4- Insatisfactorio: Más del 15\% de la ZT no visible debido a factores anatómicos, por lo demás negativo; y 5- Defectuoso: El cervigrama no puede ser evaluado por problemas de la cámara, flash, lago de ácido acético, moco, etc.

Los cervigramas fueron evaluados por el autor quien es colposcopista con 7 años de experiencia, los resultados de la CV no estuvieron disponibles al evaluar los cervigramas.

La evaluación de las CV fue realizada por citotecnólogas quienes desconocían los resultados de los cervigramas, aplicando la clasificación de Papanicolaou.

Las pacientes con CV Pap II con atipias coilocíticas, Pap III o mayor, así como las pacientes con cervigramas positivos, fueron remitidas a colposcopia donde se tomaron biopsias dirigidas $(\mathrm{Bx})$. Las pacientes con cervigramas negativos o atípicos y con $\mathrm{CV}$ representativa del exo y endocérvix dentro de límites normales no recibieron estudio adicional.

\section{Análisis estadístico}

Se definió como caso positivo aquel con diagnóstico histológico, a partir de $\mathrm{Bx}$ dirigida por colposcopia, de infección por VPH, NIC o cáncer. El grado de concordancia entre el diagnóstico histológico y el citológico no se consideró.

Se definió como caso negativo: 1- Casos con cervigrama negativo o atípico y CV Pap I ó Pap II representativa; 2- Dos set de Bx múltiples dirigidas por colposcopia negativas en casos con $\mathrm{CV}$ positiva o cervigrama positivo.

Para el análisis estadístico se tuvieron en cuenta los informes iniciales de CV y de anatomía patológica (AP). La correlación de resultados se realizó valorando la sensibilidad y especificidad de lo métodos de screening (CV y cervitografía). Sensibilidad $=\mathrm{VP} / \mathrm{VP}+\mathrm{FN}$; especificidad $=$ VN/VN+FP; $\mathrm{V}=$ verdadero, $\mathrm{P}=$ positivo, $\mathrm{F}=$ falso, $\mathrm{N}=$ negativo, mediante la prueba de proporciones se evaluaron las diferencias entre la sensibilidad y especificidad de la cervigrafía versus la $\mathrm{CV}$ tomando como valor crítico 0.05 .

\section{Resultados}

Se practicaron 112 cervigramas en igual número de pacientes siendo descartadas 7 por presentar cervigramas insatisfactorios defectuosos, quedando 105 pacientes con cervigramas analizables. 35 presentaro $C V$ positivas (Pap IIAC, Pap III o mayores), y 70 con CV negativas (Pap I o Pap II).

De las 35 pacientes con $\mathrm{CV}$ positivas, 29 tuvieron cervigramas positivos (hallazgos confirmados con $\mathrm{Bx}$ ); 5 pacientes con cervigramas atípicos: 1 caso con CV Pap IIAC (con Bx positiva), 3 casos con CV Pap IIIDL ( 2 de estos casos con $\mathrm{Bx}$ positiva y uno con $\mathrm{Bx}$ que mostró metaplasia inmadura atípica) y 1 caso con CV Pap IIIDS (con Bx positiva); $y$ un caso con cervigrama negativo: $\mathrm{La} \mathrm{CV}$ correspondiente fue informada como A3B2 DL, Bx múltiples informaron cervicitis crónica, CV de control Pap II.

En las 41 pacientes con cervigramas positivos, 6 presentaron CV Pap II. En estos 6 casos la Bx mostró: 3 metaplasia escamosa, 1 hiperplasia microglandular, 1 pólipo endocervical y 1 caso fue informado como cérvix normal); 7 casos con CV Pap IIAC, 3 casos con CV Pap IIIDL, 6 casos con CV Pap IIIDM y 13 casos con CV Pap IIIDS. En todos estos casos de CV positiva el estudio histológico confirmó patología de infección por VPH y/o NIC); y 6 casos con CV Pap II y AP con condiloma plano o NIC (Tabla 1).

\section{Tabla 1}

CERVIGRAMAS POSITIVOS Y CV PAP II (NO SE INCLUYEN CV PAP IIAC). DIAGNOSTICO AP MAS IMPORTANTE

\begin{tabular}{|ll|}
\hline Condiloma plano: & 3 \\
Displasia leve: & 1 \\
Displasia moderada: & 1 \\
Displasia severa: & 1 \\
\hline Total & 6 \\
\hline
\end{tabular}

En el presente estudio hay 39 casos positivos. La cervigrafía tuvo VP 35, FN 4, FP 6 y VN 60.

La CV tuvo VP 33, FN 6, FP 2 y VN 64.

La sensibilidad de la cervigrafía y de la CV para detectar lesiones significativas se presenta en la Tabla 2 en donde se muestra además la sensibilidad de otros estudios similares. Se incluyen los valores de la especificidad de la cervigrafía y de la CV comparándolos con los de otros estudios.

Tabla 2

SENSIBILIDAD Y ESPECIFICIDAD DE LA CV Y DE LA CERVIGRAFIA PARA DETECTAR INFECCION POR VPH O NIC

\begin{tabular}{|l|l|}
\hline Cervigrafía & Citología \\
\hline Sensibilidad $89 \%$ & $52 \%$ (Kesic, ref. 22) \\
$83 \%$ & $42 \%$ (Miloiu, ref. 23) \\
$90 \%$ & $68 \%$ (Campion, ref. 20) \\
$90 \%$ & $84 \%$ (Presente estudio) \\
Especificidad 92\% & $94 \%$ (Kesic) \\
$95 \%$ & $98 \%$ (Miloiu) \\
$92 \%$ & ND (Campion) \\
$91 \%$ & $97 \%$ (Presente estudio) \\
\hline
\end{tabular}




\section{Discusión}

Al probar las diferencias de proporciones entre la cervigrafía y la CV se encontró que no hay diferencia significativa en la sensibilidad $(p=0.2483)$ ni en la especificidad ( $p=0.0721)$ de la cervigrafía con respecto a la CV. Los resultados del presente trabajo confirman los publicados en la literatura internacional en el sentido que la cervigrafía es tan válida como la CV en la detección del NIC (24). La cervigrafía fue superior a la CV para la detección de lesiones por papiloma virus. Sólo el 10 al $30 \%$ de las infecciones por VPH pueden diagnosticarse en el frotis citológico (16). Se ha informado que la cervigrafía detecta 3 veces más pacientes con VPH que la sola CV (12). La ventaja de la cervigrafía sobre la CV en la detección del VPH tiene gran importancia clínica ya que la diferencia histológica entre la infección subclínica por VPH (o condiloma plano) y el NIC I es subjetiva. Es mejor clasificar las lesiones como de "Bajo grado" (incluyen infección por VPH y NIC I) y de "Alto grado" (incluyen NIC II - NIC III, CA in situ), tal y como lo propone la clasificación de Bethesda (17).

\section{Conclusiones}

El uso simultáneo de la CV y la cervigrafía mejora la detección del NIC y del VPH, disminuye el número de pacientes que tendrían que ser remitidas a colposcopia (todas aquellas Con CV Pap II persistente, atipias severas, atipias coilocíticas, Pap III o superior) y permitiría disminuir la frecuencia de la toma de CV lo que compensa con creces el costo adicional (el costo de la cervigrafía es similar al de una CV según la tarifa de la Sociedad Colombiana de Patología), quedando además un registro gráfico del cérvix, documento de gran valor no sólo desde el punto de vista clínico sino docente e investigativo.

\section{BIBLIOGRAFIA}

1. Coppelson LW., Brown B. Estimation of the screening error rate from observed detection rates in repeated cervical cytology. Am. J. Obstet. Ginecol. 1974; 119: 953.

2. Fetherston WC. False-negative cytology in invasive cancer of the cervix. Clin. Obstet. Gynecol. 1983; 26: 929.

3. Day NE. Effect of cervical cancer screening in Scandinavia. Obstet. Gynecol. 1984; 63: 714 .

4. Stenkvist B., Bergstrom R., Eckland G. et al. Papanicolaou smear screening and cervical cancer: what can you expect? JAMA 1984; 252 : 1423.

5. Burk R., Kadish AS., Calderin S., Romney SL. Human papillomavirus infection of the cervix detected by cervicovaginal lavage and molecular hybridization: correlation with biopsy results and Papanicolaou smear. Am. J. Obstet. Gynecol. 1986; 154: 982.

6. Jones Ded., Craeasman WT., Dombroski RA., Lentz SS., Waeltz JL. Evaluation of the atypical pap smear. Am. J. Obstet. Gynecol. 1987; 157: 544 .

7. Davis GL., Hernandez E., Davis JL., Miyazawa K. Atypical squamous cells in papanicolaou smears. Obstet. Gynecol. 1987; 69: 43.

8. Hulka BS. Cytologic and histologic outcome follewing and atypical cervical smear. Am. J. Obstet. Gynecol. 1968; 101: 190.

9. Spitzer M., Krumholtz BA., Seltzer LA., Molho L. Cervical cancer detected by cervicography in a patient with negative cervical cytology. Obstet. Gynecol. 1986; 68: 68S.

10. Himmelstein LR. Evaluation of inflammatory atypia: A literature review. J. Reprod. Med. 1989; 34: 634.

11. Pearlstone AC., Grigsby PW., Mutch DG: High rates of atypical cervical cytology: Occurrence and clinical significance. Obstet. Gynecol. 1992; 80: 191.

12. Rehder KE., Blythe JG. Cytology and cervicography compared to cytology alone for human papillomavirus detection. Colposcopy \& Gynecologic Laser Surgery 1988; 4: 1.

13. Soutter WP., Wisdom S., Brough AK., Monaghan JM. Should patients with mild atypia in a cervical smear be referred for colposcopy? Brit. J. Obstet. Gynecol. 1986; 93: 70.
14. Stalf A. Cervicography: A new method for cervical cancer detection. Am. J. Obstet. Gynecol. 1981; 41: 168.15. Tawa K., Forsythe A., Cove JK., Saltz A., Peters HW., Watring WG. A comparison of Papanicolaou smear an the cervigram: sensitivity, specificity, and cost analysis. Obstet. Gynecol. 1988; 71: 229.

16. Schneider V. Diagnóstico microscópico de infección por papilomavirus humano. Clin. Obstet. Gynecol. 1989; 1: 143.

17. National Cancer Institute Workshop. The 1988 Bethesda System for reporting cervical/vaginal cytological diagnoses. JAMA 1989; 262: 931.

18. Urdaneta H., Amortegui A. The prevalence of human papillomavirus genotypes in cervical intreepithelial neoplasia and cervical cancer in Bogota, Colombia. En publicación.

19. Reid R. Human papillomaviral infection. The key to rational triage of cervical neoplasia. Obstet. Gynecol. Clin. North. Am. 1987; 14: 407.

20. Campion MJ., Di Paola FM., Vellios F. The values of cervicography in population screening. 7th World Congress of Cervical Pathology and Colposcopy, Rome, 1990.

21. Di Paola FM., Campion MJ., Echemendia MM., Rothrock R., Franklin EW., Velloios F. Moderm approach to triage af atypical cervical citology. 7th World Congress of Cervical Pathology and Colposcopy, Rome, 1990.

22. Kesic V., Soutter WP., Juznic N., Aleksic M., Ljubic A., Sulovic V. A comparison of colposcopy, cytology and cervicography in screening. 7 th World Congress of Cervical Pathology and Colposcopy, Rome, 1990.

23. Miloiu A., Guenier C., Andry M., Hertens D., Bonnal JL., de Launoit Y., Nogaret JM., Coibion M. Correlation between the pap smear and a new screening approach: The cervicography. The Belgian experience. 7th World Congress of Cervical Pathology and Colposcopy, Rome, 1990.

24. Reid R., Zado B., Husain M., Jenson AB., Lorinz AT., Daoud Y., Laverty CR. Should cervical cytology by augmented by cervicography or HVP DNA detection? 7th World Congress of Cervical Pathology and Colposcopy, Rome, 1990.

25. Stalf A. Cervicografía. Clin. Obstet. Ginecol. 1983; 4: 1223. 